\title{
Studies of lactic dehydrogenase content in rectal mucosal biopsies
}

\author{
P. S. HUNT, P. M. DENNIS, P. JABLONSKI, S. K. KHOO, L. MCLEOD, \\ H. D. P. THOMSON, AND J. MCK. WATTS \\ From the Department of Surgery, Monash University Pathology Services, Prince Henry's Hospital, \\ Melbourne, and Department of Obstetrics and Gynaecology, University of Queensland, Brisbane, \\ Australia
}

SUMMARY The total lactic dehydrogenase (LD) content and LD isoenzyme ratios were studied in homogenized rectal mucosa from 31 patients with established non-specific mucosal ulcerative colitis and from 16 normal subjects. The total LD content was found to be significantly increased in patients with active ulcerative colitis when compared with patients with inactive colitis or with normal subjects. There was a similar though not a significant increase in the slow moving isoenzymes of LD in samples of rectal mucosa from patients with active colitis. The LD isoenzyme pattern was in the normal range in two of the three patients with histological premalignant changes in rectal biopsy specimens.

There was a statistically significant linear correlation between the total lactic dehydrogenase content of rectal mucosa and the carcino-embryonic antigen levels in whole serum.

Both the total content and isoenzyme ratios of LD were increased in neoplastic tissue obtained from patients with carcinoma of the colon and with tissue from benign rectal polyps.

Morson and Pang (1969) suggested that a search for cellular atypia in rectal biopsies is a valuable method for detecting those patients with chronic ulcerative colitis susceptible to colo-rectal cancer. They considered these changes to represent premalignancy, and in further studies (Lewis, Morson, February, Hywel-Jones, and Misiewicz, 1971) regularly detected an abnormal lactic dehydrogenase (LD) isoenzyme pattern in homogenates of such rectal biopsy specimens.

This study is concerned with an analysis of total LD content and the pattern of its isoenzymes in homogenates of rectal mucosa obtained from patients with ulcerative colitis and neoplastic disease of the colo-rectum. The whole sera of these patients were also tested for the presence of carcino-embryonic antigen (CEA) by a microradioimmunoassay (Khoo and MacKay, 1973).

\section{Patients and Methods}

This study included 31 patients with the clinical, radiological, and histological features characteristic of non-specific mucosal ulcerative colitis, according

Received for publication 11 September 1974. to the criteria defined by Morson (1969). Seventeen of these 31 patients had a previous colectomy and ileo-rectal anastomosis. A further distinction was made on clinical and sigmoidoscopic grounds into those with active rectal disease (14 patients) and those with mild or inactive disease (17 patients). Eight of 11 patients with chronic ulcerative colitis and six of 10 patients after ileo-rectal anastomosis were considered to have active rectal disease. The age range of patients was from 21 to 72 years with 16 males and 15 females. The duration of disease varied from one month to 21 years with a mean duration of 71 months (standard deviation 74 months). The duration of disease in the 17 patients operated upon was $112 \pm 70$ months; the time since operation was $65 \pm 40$ months. Synchronous studies were performed in 16 age- and sex-matched subjects without colitis or neoplastic disease and with histologically normal rectal mucosa. Neoplastic tissue from five patients with carcinoma of the large bowel and six patients with benign rectal polyps was also studied.

All the biopsy specimens were examined histologically without knowledge of the clinical status of the patient for the presence of premalignant changes according to the criteria defined by Morson (1969) 
They were also analysed histologically for disease activity. The histological assessment of activity invariably agreed with the assessment made clinically on macroscopic appearances.

Mucosal sections with a weight range of 8 to $50 \mathrm{mg}$ were homogenized in 0.2 to $0.3 \mathrm{ml}$ of cold physiological saline in a glass Potter-Elvehjem homogenizer and LD was estimated by an autoanalyzer method (Technicon manual). This homogenate was stored at $4^{\circ} \mathrm{C}$ and assayed within 24 hours of the biopsy. Pilot experiments, using rectal mucosa obtained from three patients with ulcerative colitis and one patient with carcinoma of the colon, indicated that the total LD activity of the homogenate fell with storage and that the LD isoenzyme pattern was changed. The isoenzymes of LD were separated by electrophoresis at $\mathrm{pH} 8.6$ (barbital buffer, ionic strength $=0.075$ ) on cellulose acetate strips. The isoenzymes were detected by the production of a blue formazan on incubation of the strips with $0.1 \mathrm{M}$ sodium lactate $/ 1 \%$ NAD $/ 0 \cdot 1 \%$ MTT tetrazolium/ $0.1 \%$ phenazine methosulphate (Barnett, 1964). The stained strips were dried in air and cleared with ondina 17 oil (Shell) before scanning at 520 using an integrating densitometer. A ratio was calculated as follows:

$$
\frac{\text { LD } 4+\text { LD } 5}{\text { LD } 1+\text { LD } 2} \text {. }
$$

Whole serum CEA was measured by a microradioimmunoassay. Whole sera containing more than $3.0 \mathrm{ng} / \mathrm{ml}$ of CEA were extracted with perchloric acid and reanalysed (Khoo, Hunt, and MacKay, 1973).

\section{Results}

In this study variation from control results in total
LD content and isoenzyme ratio in rectal mucosa was not related to the duration of disease or whether the patient had been previously subjected to subtotal colectomy and ileo-rectal anastomosis. Despite a clinical duration of colitis in some patients up to 21 years, only three patients were found to have evidence of cellular atypia and polypoid changes in mucosal sections. From two of these three patients rectal mucosa had a total LD and isoenzyme ratio within the normal range (figs 1 and 2). The third patient also had histological and clinical evidence of active disease which could have produced the increased total LD content and isoenzyme ratio found (figs 1 and 2). This patient also had a slightly raised whole serum CEA level of $3.5 \mathrm{ng} / \mathrm{ml}$, which was extractable with perchloric acid.

Increased total LD content was directly related to the activity of disease in the rectum whether from those patients with an intact colon or from those with a previous ileo-rectal anastomosis. The total LD in rectal mucosa of those 14 patients with active disease was $105 \pm 27 \mathrm{IU}^{1}$ (International Units/g wet weight). This value was significantly greater than the total LD content of $20 \pm 13 \mathrm{IU} / \mathrm{g}$ wet weight in 16 normal subjects and $44 \pm 17 \mathrm{IU} / \mathrm{g}$ wet weight in the 17 patients with mild or inactive disease $(P<0.01)^{2}$ (fig 1). The neoplastic tissue homogenate from six patients with carcinoma of the colon contained $105 \pm 27 \mathrm{IU} / \mathrm{g}$ wet weight of LD activity and this was also significantly greater than the total LD content in the rectal mucosa of normal subjects (P < 0.01) (fig 1) but no different from that obtained from patients with active colitis.

The results obtained from analysis of LD iso-

${ }^{1}$ All values given are mean \pm 1 standard deviation.

'Statistical analyses by Wilcoxon's sum of ranks test.

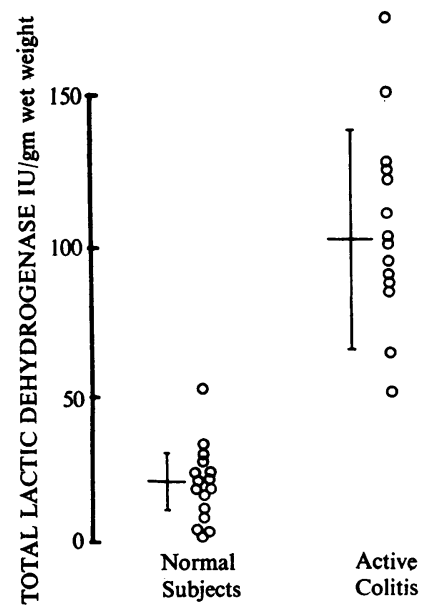




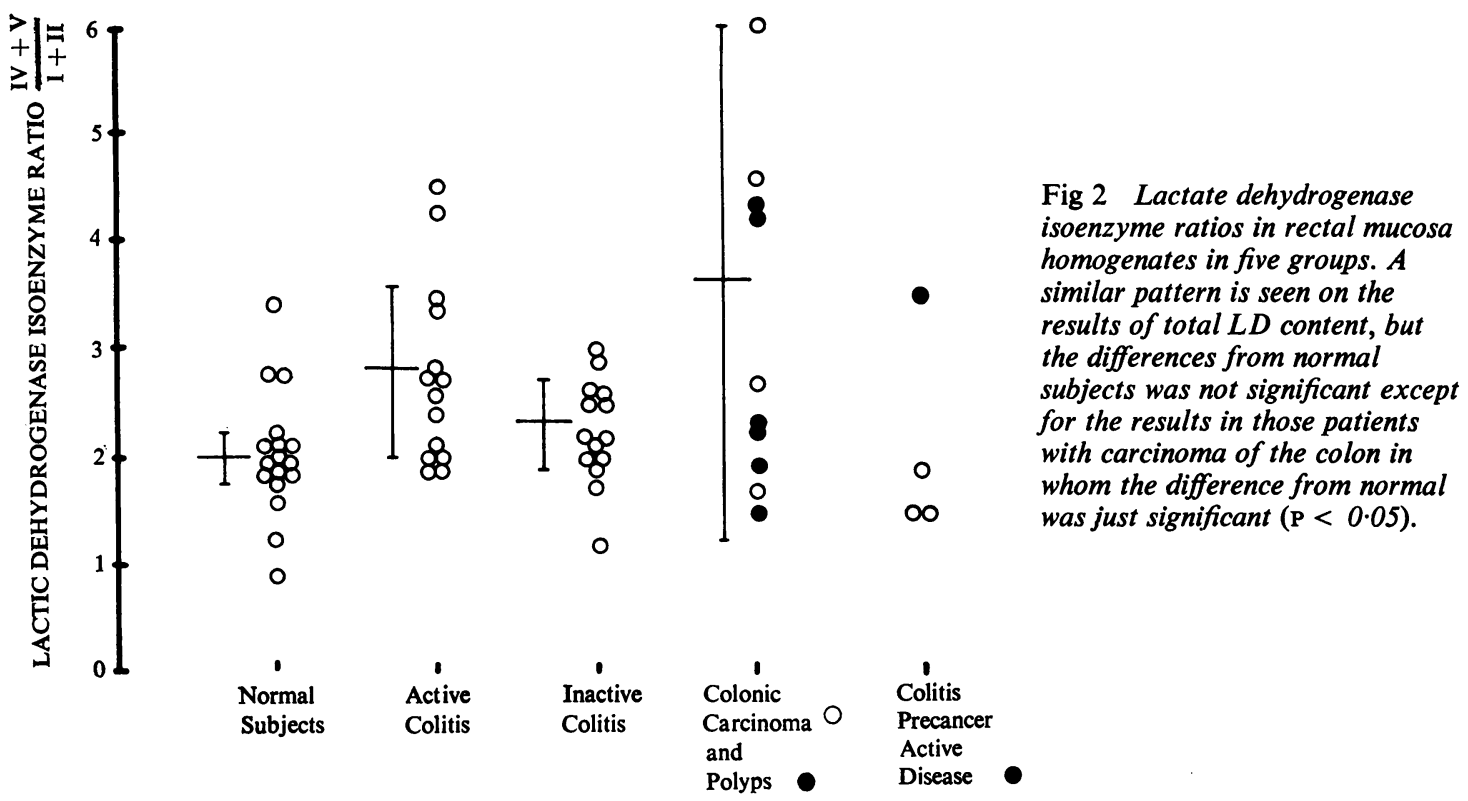

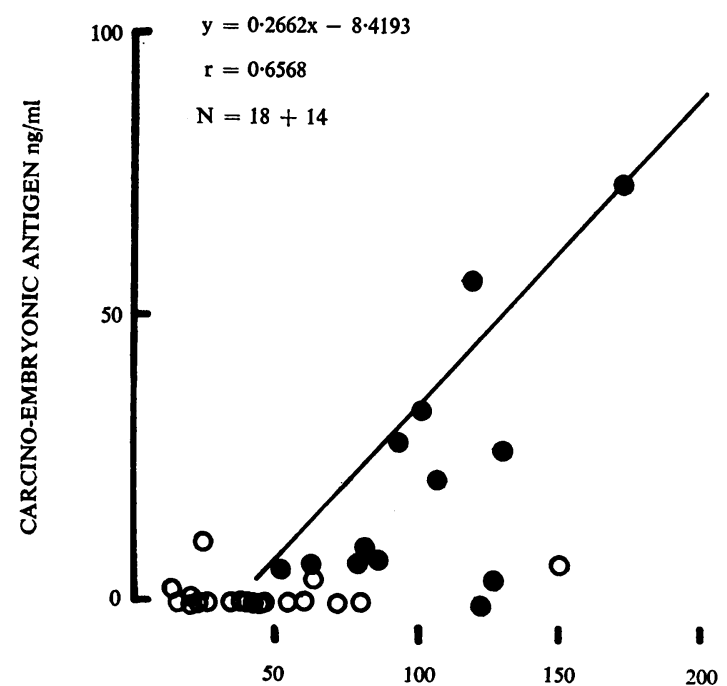

TOTAL LACTIC DEHYDROGENASE IU/g

Fig 3 The linear correlation between mucosal total $L D$ content and the carcino-embryonic antigen levels in whole $\operatorname{serum}(r=0.6568, \mathrm{P}<0.01) . \mathrm{O}=$ Inactive colitis; $=$ active colitis. enzyme ratios showed a similar trend to the results for total LD content, but the difference from ratios in normal subjects was not significant at the 5\% level of probability (fig 2). The LD isoenzyme ratio was $2.8 \pm 0.8$ in active colitis, $2.3 \pm 0.3$ in inactive colitis, and $2 \cdot 0 \pm \mathbf{0 . 2}$ in normal subjects (fig 2). The isoenzyme ratio in some neoplastic tissue was considerably elevated and the difference from normal rectal mucosa was just significant at the $5 \%$ level (fig 2).

It has already been shown that whole serum CEA levels are raised in active colitis and carcinoma of the colon (Khoo, Hunt, and MacKay, 1973). This study also showed that CEA reactivity was again extractable with perchloric acid in active colitis, but not in carcinoma of the colon (Khoo et al, 1973).

There was a statistically significant linear correlation between the total LD content of rectal mucosa and the CEA levels in whole serum. In patients with active disease both the parameters measured were elevated (fig 3). Six patients were studied during an active phase of the disease and later during remission following treatment by either topical or systemic corticosteroids. In five patients there was a fall in the total LD content of rectal mucosa and in all patients a fall in whole serum CEA levels with clinical 


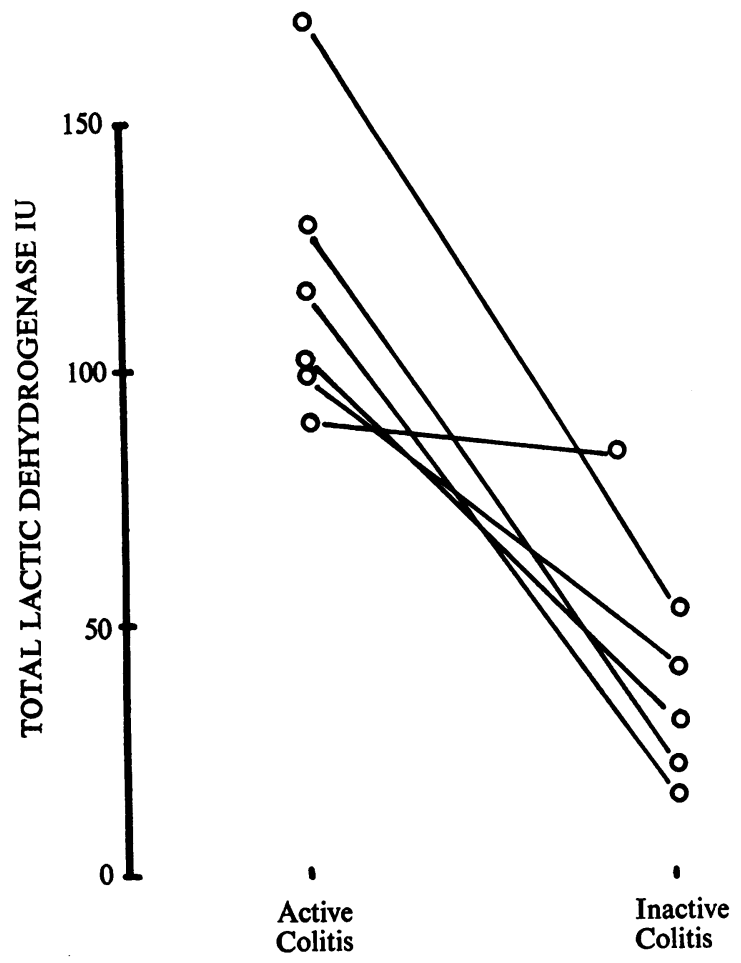

Fig 4 Six patients were studied during an active phase of colitis and later during remission. In five patients there was a fall in total $L D$ content of rectal mucosa to within the normal range corresponding with clinical remission of disease.

remission (figs 4 and 5). These results confirm the observations already made between the two groups of patients with different activity of disease.

\section{Discussion}

In this study the LD content in rectal mucosal samples was found to be related to the clinical activity of the ulcerative colitic process. This finding correlates with increased whole serum CEA levels in patients with active colitis. These increases occurred whether the disease was confined to the rectal mucosa, as found after ileo-rectal anastomosis, or whether the patients studied had an intact colon. Thus, an increase in LD content or isoenzyme ratio does not necessarily indicate premalignancy, implicit in the findings of Lewis and his colleagues (1971). Although only three patients in this group were considered to have histological evidence of premalignancy only one had an increased LD content and an LD isoenzyme ratio. This patient also had macroscopically and microscopically active disease. Such variations in $3^{*}$

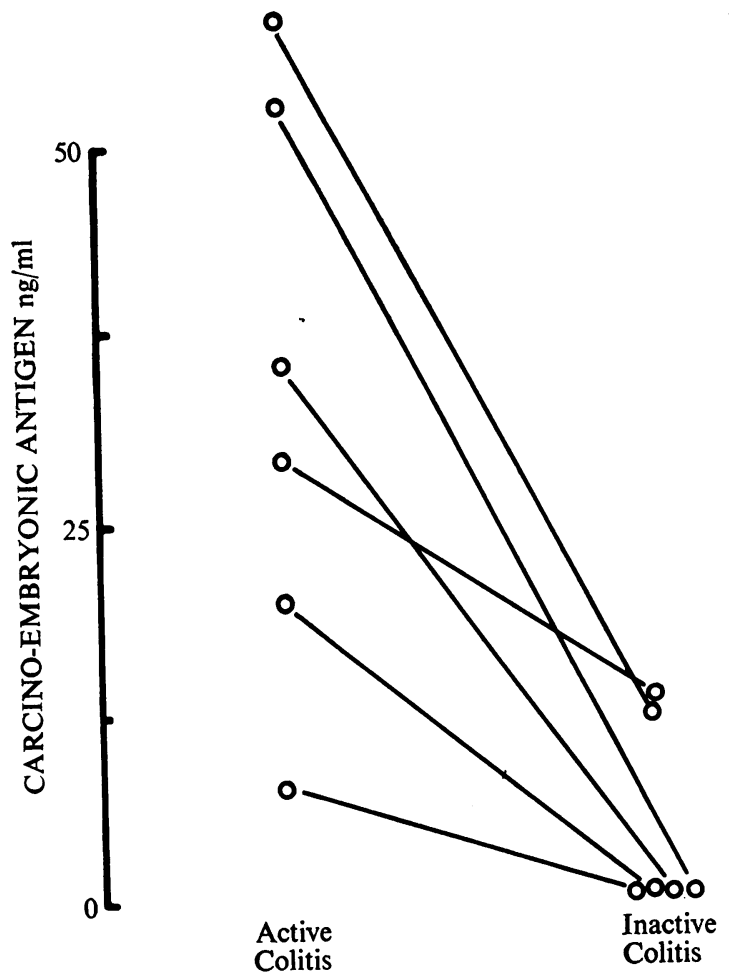

Fig 5 The whole serum carcino-embryonic antigen in six patients during an active phase of colitis and later during remission. There was a fall in each patient after clinical resolution of disease.

LD content cannot therefore be considered a reliable index of premalignancy and may thus be of doubtful clinical usefulness in the early detection of colonic carcinoma. It should be noted that assay conditions used in this study were different from those used by Lewis and his colleagues (1971) and that this may account for the discrepancy in our data for the control group. Any procedure to assay total LD activity is at best a measure of the weighted sum of the activities of each of the isoenzymes present in the specimen and the activity of each is a function of $\mathrm{pH}$, substrate, and NAD concentrations. Both assays used lactate as the substrate but concentrations and $\mathrm{pH}$ were dissimilar. In this study $\mathrm{pH} 8.6$ was used rather than 10.00. This $\mathrm{pH}$ has been shown (Gay, McComb, and Bowers, 1968) to be in the $\mathrm{pH}$ range for minimal variation due to the isoenzyme make up of the specimen and that at least $96 \%$ of the activity of $\mathrm{LD} 1,3$, and 5 was measured at this $\mathrm{pH}$.

The separation of isoenzymes differed in that the supporting medium was cellulose acetate rather than agar. The method used was essentially that of Barnett 
(1964), and has been validated (Barnett, 1964; Gay et al, 1968), who showed that recovery of LD activity from cellulose acetate was complete. Isoenzyme ratios obtained by us for tissues (liver, kidney, heart, skeletal muscle) and serum (normal and postmyocardial infarct) using cellulose acetate were similar to those reported using agar. The use of cellulose acetate has the theoretical advantage of its higher porosity which enables better mixing of enzyme and substrate. The assay of isoenzymes after electrophoresis was similar in both studies. Both assay media were used in pilot studies and identical isoenzyme ratios were obtained.

In this study the neoplastic tissue from patients with colon carcinoma and benign rectal polyps contained a significantly increased content of $L D$ and an increased LD isoenzyme ratio. It is possible that abnormally high values of LD are closely related to increased cell turnover in active colitis (Eastwood and Trier, 1973) and perhaps also in carcinoma. It may also be an adaptation to conditions of oxygen deficit. Glycolysis is known to be increased in neoplastic tissue, possibly as an adaptation to anaerobic conditions. The high glycolytic activity of tumour tissue was reported as long ago as 1923 by Warburg and Minami (1923). Goodfriend, Sokol, and Kaplan (1966) have shown that exposure of cells in tissue culture to low oxygen tensions provokes increased synthesis of LD 5. Langvad (1968) found that a shift to a preponderance of LD 4 occurred in colonic tumours and in the surrounding tumour-negative areas. He interpreted this to be a metabolic change in cells which may subsequently be malignant and it was proposed that these tumours develop in tissue which has undergone a shift to increased glycolysis. The increased LD content may thus be an expression of a similar oxygen deficit in the mucosal cells of patients with acute ulcerative colitis rather than an index of premalignancy.

We should like to thank Professor E. S. R. Hughes and Doctors J. Hansky, M. Korman, H. Hillman, and G. Schmidt for the opportunity to study their patients.

References

Barnett, H. (1964). The staining of lactic dehydrogenase isoenzymes after electrophoretic separation on cellulose acetate. J. clin. Path., 17, 567-570.

Eastwood, G. L., and Trier, J. S. (1973). Epithelial cell renewal in cultured rectal biopsies in ulcerative colitis. Gastroenterology, 64, 383-390.

Gay, R. J., McComb, R. B., and Bowers, G. N., Jr. (1968). Optimum reaction conditions for human lactate dehydrogenase isoenzymes as they affect total lactate dehydrogenase activity. Clin. Chem., 14, 740-753.

Goodfriend, T. L., Sokol, D. M., and Kaplan, N. O. (1966). Control of synthesis of lactic acid dehydrogenases. J. molec. Biol., 15, 18-31.

Khoo, S., Hunt, P. S., and Mackay, I. R. (1973). Studies of carcinoembryonic antigen activity of whole and extracted serum in ulcerative colitis. Gut, 14, 545-548.

Khoo, S., and Mackay, I. (1973). Carcino-embryonic antigen in serum in diseases of the liver and pancreas. (Submitted for publication.)

Langvad, E. (1968). Lactic dehydrogenase isoenzyme patterns in the tumour bearing colon. Int. J. Cancer, 3, 17-29.

Lewis, B., Morson, B. C., February, A. W., Hywel-Jones, J., and Misiewicz, J. J. (1971). Abnormal lactic dehydrogenase isoenzyme patterns in ulcerative colitis with precancerous change. Gut, 12, 16-19.

Morson, B. C. (1969). Pathology. In Ulcerative Colitis, edited by J. C. Goligher, F. T. de Dombal, J. M. Watts, and G. Watkinson, pp. 5-31. Baillière, Tindall, and Cassell, London.

Morson, B. C., and Pang, L. S. C. (1969). Rectal biopsy as an aid to cancer control in ulcerative colitis. Gut, 8, 423-434.

Technicon AutoAnalyzer. Method N-60.

Warburg, O., and Minami, S. (1923). Versuche an überlebendem carcinomgewebe. Klin. Wschr., 2, 776-777. 